\title{
Ética y gerencia universitaria
}

\author{
Guijarro de Chávez, Mayra* \\ Chávez Sánchez, Jorge**
}

\section{Resumen}

En el mundo de hoy, cada vez adquiere mayor relevancia la necesidad de una conducta ética en la gestión de las universidades que contribuya a asumir prácticas gerenciales congruentes en los distintos intereses que coexisten en el entorno académico. De ahí, que este trabajo se plantea a través de una labor documental, la revisión de la gerencia y organización de la universidad, asumiendo un enfoque sustentado en la necesidad de los principios éticos como ordenadores del nuevo esquema que prevalezca en las instituciones de Educación Superior Venezolanas en el siglo $X X I$. Los resultados apuntan a señalar que el papel de la Universidad Venezolana en este momento de cambio necesario y fundamental, representa una nueva expresión de la forma de vida del universitario, lo cual resulta una responsabilidad ineludible y prioritaria como venezolanos. El gran desafío de la Universidad Venezolana es proveer educación de calidad y el deber ético mas importante de esta institución es iluminar a los estudiantes, demostrar que las sombras son sombras y que hay que recorrer el camino hacia la luz.

Palabras clave: Gerencia, ética, universidad, valores.

\section{Recibido: 06-04-05 Aceptado: 06-04-06}

Doctora en Ciencias Gerenciales, Profesora Titular en la Facultad de Ciencias Económicas y Sociales, LUZ. Maracaibo-Venezuela. E-mail: guijarromayra@yahoo.com.

** Doctor en Ciencias Gerenciales. Investigador acreditado en el Programa de Promoción al Investigador (PPI No. 4.801). Maracaibo-Venezuela. E-mail: jechavez@luz.edu.ve. 


\section{Ethics and University Management}

\section{Abstract}

In the world today, the need for an ethical conduct is ever more relevant in the management of universities and this contributes to the need to assume congruent management practices in the distinct areas that co-exist within the academic world. This paper proposes a documentary review of university management and organization from a perspective based on the need for ethical principles as the pattern for a new framework which should prevail in Venezuelan institutions of higher learning in the XXI century. The results point out the role of the Venezuelan university in this moment of necessary and fundamental change, representing a new expression of a new form of university life, which turns out to be an unavoidable and primary need for Venezuelans. The great challenge of Venezuelan universities is to provide quality education and ethical institutional responsibility in order to illumine students and to demonstrate that shadows are shadows, and we must choose the pathway to light.

Key words: Management, ethics, university, values.

\section{Introducción}

La Universidad en Venezuela como espacio multidimensional y complejo, asiste a una gama de interesantes posibilidades para lograr procesos profundos de cambios y transformaciones dinámicas consustanciales a su propia naturaleza.

En ese sentido, las casas de estudios superiores se encuentran inmersas en un mundo permeable en el cual las diferentes posiciones teóricas influyen de manera importante, encontrando en el interior de estas organizaciones condiciones apropiadas para actuar en razón de sus actividades principales: docencia, investigación, extensión y producción; llevando a convertirlas en un espacio sin fronteras comunicacionales, donde el dominio del conocimiento ayuda a la concreción de sociedades del saber (Ramírez et al, 2002).

Esta primacía de funciones en las organizaciones educativas universitarias estimula a las mismas y a sus miembros, a la auto-organización y la interrelación de sus diferentes componentes con el objetivo de cumplir con sus funciones ante la sociedad.

Sobre el particular, las Universidades tienen la responsabilidad de crear y colocar a disposición de la sociedad saberes pertinentes, tal como lo señalan González et al, (2002), para estar así a la vanguardia en el tratamiento de temas políticos y sociales desde la perspectiva de una organización con un gobierno éticamente responsable, como clave para incidir en las transformaciones de su interioridad, pero también de la sociedad, con un sentido de compromiso social basado en la justicia manifestada por la toma de decisiones de sus actores.

Lo crítico es cómo hacer entender que la idea de cambio, por intermedio de una conciencia ética posicionada en el centro de la organización sea aceptada y comprendida como un compromiso de la institución y de sus líderes.

Se pretende en este caso, a partir de la organización universitaria y de su 
gerencia, buscando en la ética, acercar ese mundo de valores al gobierno universitario e insertarlo como una necesaria fórmula que conduzca hacia los cambios de la universidad venezolana, partiendo por su ubicación como elemento clave en la responsabilidad social y en la toma de decisiones.

De ahí, que este trabajo se plantea a través de una labor documental la revisión de la gerencia y organización de la Universidad, tomando como base primaria el medio venezolano, asumiendo un enfoque sustentado en la necesidad de los principios éticos como ordenadores del nuevo esquema que prevalezca en las instituciones de educación superior venezolanas en el siglo XXI.

\section{La Gerencia dentro de un nuevo paradigma organizacional}

En los últimos treinta años, las sociedades han abierto nuevos espacios, de ahí que otras exigencias y escenarios se han hecho presentes (Giddens, 2003). Hay un ambiente de velocidad constante. El hombre emerge de un contexto revolucionado por la tecnología; los ambientes sociales, económicos, políticos y organizacionales están llamados a transformarse y adaptarse a las exigencias de este nuevo contexto.

Las organizaciones se han transformado en centros inteligentes, convirtiéndose así en mundos complejos donde los cambios y su proyección se hacen necesarios en el ambiente donde se desarrollan para así sostener su vigencia. Los cambios, avances y transformaciones de sus procesos dentro de las instituciones o empresas públicas y privadas conforman una secuencia de acciones para la presencia y dinamismo que puedan tener en el contexto social donde actúan.

En este sentido, Gibson et al, (2001) definen la organización como una unidad coordinada de esfuerzos, constituida de por lo menos dos personas, quienes trabajan hacia una meta o metas en común. De igual manera, se trata de una entidad que le permite a la sociedad perseguir logros que no se pueden obtener por individuos actuando solos.

En efecto, el nuevo escenario ha llevado a las instituciones públicas, privadas con o sin fines de lucro, a modificar -a lo largo de las últimas décadas del siglo $X X$ - sus procesos y estructuras organizacionales de acuerdo con diferentes modelos de comportamiento organizacional, con la finalidad de adaptarlas a las características y exigencias de los cambios paradigmáticos manifestados a partir de la sociedad de la información y del conocimiento (Gibson et al, 2001).

Por esta razón, uno de los aspectos fundamentales de toda organización institucional, es la ejecución de la gerencia que se requiere de acuerdo a las nuevas exigencias. Esto implica la puesta en escena de procedimientos administrativos, gestión e innovación de la dirección con un liderazgo adecuado al contexto social, económico y político, así como una actitud ética sostenida y permanente conducente al bien común, como integrador de lo humano en bien de la comunidad (Sánchez, 2002).

La gerencia requiere movilidad y una dinámica constante dentro del tiempo y espacio universal, a objeto de cumplir con la revolución para las cuales es- 
tán llamadas las organizaciones, con la búsqueda de la eficiencia y de una imagen de excelencia y productividad en un contexto de eticidad (Drucker, 2003).

Al respecto, Giddens (2003) afirma que la década de los '70 en adelante se considera como la época de la cultura organizacional donde aparecen enfoques, modelos y paradigmas innovadores para rebatir la gerencia tradicional y formal. Es en este tiempo cuando se inicia un camino para un nuevo liderazgo gerencial que implica la participación de la gente, la modernización de los procesos, la innovación, la equidad, la calidad y la producción de bienes sociales.

De igual forma, Desiato y Guevara (1998) señalan que las organizaciones deben ser transformadas, ese es el ideal, pero este cambio debe partir de su propio centro de gravedad, de su evaluación, en el cual la revisión interna debe experimentarse en razón de su funcionamiento, su dimensión, pero asimismo, de su objetivo frente a la sociedad en la cual está inmersa.

Es así como se visualiza la organización movida por procesos conformes a su configuración, en la cual la toma de decisiones debe ser emplazada a los procesos gerenciales que orienten los mismos cambios sustentados en su dinamismo, siendo el entorno de manera decidida, un espacio donde se produce una gran influencia hacia el interior de las organizaciones, el cual no permite olvidar los valores que deben prevalecer en la ellas (Castells, 1999).

En otro orden de ideas, Cortina (2000) al referirse al papel de los gerentes o directivos dentro de las empresas y/o instituciones, argumenta que éste se hace cada vez más exigente y especial, porque constituye de alguna manera, conjuntamente con todo el personal, la imagen de la cultura organizacional y una de las partes esenciales de la institución, donde el directivo se ha convertido en uno de los personajes más significativos de la cultura de fin de siglo.

En virtud de lo anterior, las Instituciones de Educación Superior, específicamente las Universidades, deben modificar sus esquemas gerenciales con la finalidad de alcanzar calidad, eficiencia, eficacia, pertinencia, excelencia, equidad y producción de bienes sociales, que permitan establecer relaciones entre las asignaciones presupuestarias y el cumplimiento de las funciones básicas de las casas de estudios superiores, como: docencia, investigación, extensión, producción, de acuerdo a los niveles exigidos y demandados por la sociedad.

Según Castells (1999), las características del mundo social de la vida humana, se identifican como un mundo de transformaciones, cuyo motor de inicio lo constituye la tecnología de la información, la cual está modificando la base material de la sociedad a un ritmo acelerado. Además expresa que las economías del mundo se han hecho interdependientes, se derrumbó el estatismo soviético, desaparece el movimiento comunista internacional, finaliza la guerra fría, se reduce el riesgo del holocausto nuclear, se alteró la geopolítica global, el capitalismo sufre una reestructuración profunda, caracterizada por una mayor flexibilidad, las empresas se descentralizan e interconectan, se presenta un declive de las organizaciones sindicales y la mujer participa más en el mundo laboral. 
Por otro lado, aparece la integración global de los mercados financieros, el Pacífico Asiático es el nuevo centro industrial global dominante, y además, se unifica económicamente; Europa y los países del llamado Tercer Mundo se atreven a transitar por igual camino. Éste es el mundo de ahora, es el contexto y la plataforma que sustenta la vida personal, profesional y organizacional.

En esta caracterización del mundo social actual, no se obvia la necesidad de transformar el mundo organizacional de las empresas e instituciones. Hay aportes teóricos de gran importancia y utilidad los cuales como señala Drucker (2003) están señalando el camino a profundas revisiones, esquemas de una nueva sociedad del conocimiento, pero asimismo, de intereses económicos en la construcción de un nuevo orden, a fin de proyectar la nueva visión organizacional del momento, donde se pueden identificar varios de sus elementos, como: la tecnología, el aprendizaje, el hombre, el conocimiento, el cooperativismo, la pertinencia, la producción, la equidad, la calidad, la integración y con énfasis en la ética, como componente relevante y necesario.

Dentro de las transformaciones que se requieren en el mundo empresarial, se exige la puesta en práctica de una gerencia que responda a la revisión y cambios exigidos por el entorno. Esto implica el análisis de los procedimientos administrativos y de gestión para evaluar si verdaderamente el directivo está asumiendo su compromiso con la institución; además, la apertura y la innovación de una dirección con liderazgo y una actitud ética sostenida y permanente, que lleve adelante los cambios en la gerencia direccional.

La gerencia requiere movilidad y dinamismo, así como formación y capacitación de contenidos teóricos y de valores, que la lleven a visualizar los cambios requeridos y pertinentes para su entorno. La misma en sí, es el centro de las acciones para que la organización sea eficiente y refleje una imagen de excelencia y productividad. Asumir el rol de gerente, implica sostener una actuación directiva con el acertado componente de liderazgo, aunado a la ética organizacional.

No puede entenderse una gerencia que funcione con límites organizacionales entre el directivo y el líder; al respecto, Quinn (1996) señala que quien asuma el rol de gerente, debe ser director y a la vez ser líder, debe estar preparado para enfrentar grandes cambios, tanto en el aspecto administrativo-empresarial, como en las actividades que involucren el desarrollo del hombre como un ser social e integral, física y psicológicamente.

\section{Los nuevos enfoques organizacionales}

El ser un administrador-líder, implica conocer los postulados, misión y visión de la nueva administración, enfocando los esfuerzos en nuevas estrategias en la organización que le lleven a garantizar el progreso continuo del sistema donde se realizan los procesos administrativos, y en razón de las particularidades situacionales (Quinn, 1996).

Al ubicarse como administrador-líder se deben tener en cuenta de forma ecléctica los enfoques y estrategias que de acuerdo a la cultura y al clima organi- 
zacional existen en una organización. Esta situación, de conformidad con lo señalado por Muro (1998), estimula y motiva al personal a realizar los cambios pertinentes y a retener lo que es funcional dentro de la organización con un enfoque futurista en todas las actividades, de la cual no se debe exceptuar la educación.

Sobre este particular, Drucker (2003) enfoca la organización educativa hacia un futuro de alianzas y flexibilidad en la ejecución del trabajo, manteniendo propósitos unitarios en la formación de líder de líderes. El líder futurista busca ser proactivo y mantener la pertinencia en todos los niveles educativos.

Asimismo, otro enfoque administrativo que se propone es la escuela o enfoque situacional, donde Bueno (2002) propone un sistema socio-técnico con énfasis en la influencia técnica sobre el sistema social prevaleciente, relacionando lo socio-técnico con lo que surge dentro de la organización. En este sentido, la tecnología está en el sistema educativo como una herramienta de trabajo, pero la relación entre el personal y la tecnología, resulta un elemento a considerar, debiendo tener en cuenta en los estudios de necesidades, realizados en los diferentes niveles educativos del sistema y de las diferentes organizaciones que la componen, los grandes aportes que proporciona (Quinn, 1996).

Otro enfoque son "las siete eses" de McKinsey, donde Marsick (2000) revisa estos enfoques neoclásicos integrando los conceptos que establecen la excelencia organizacional (estrategia, estructura, sistema, estilo, staff, valores compartidos y habilidades), y ofrece la oportunidad al administrador de estudiar los componen- tes que tienen en cada organización, sucursal, distrito o región, y poder establecer las estrategias, procesos y enfoques efectivos de trabajo dentro del sistema del cual se trate. Esta teoría humanística y enfoque de procesos ofrece la alternativa de realizar trabajos eficientes y efectivos, al montar los procesos organizacionales dentro de su realidad social, emocional y basada en las aptitudes del recurso humano existente dentro de la organización.

Por otra parte, el enfoque técnicosocial mueve al líder a un enfoque de contingencia, como lo exponen Argyris y Shön (2001), donde el estudio de las influencias de las circunstancias sobre la estructura organizativa y el comportamiento administrativo, ofrece información valiosa sobre las estrategias funcionales y las no funcionales, lo que complementaría de manera directa el enfoque situacional, pero de forma integrada a través del enfoque de "las siete eses".

Los enfoques modernos se mueven en torno a lo operacional, de allí que Koontz y Weihrich (2002) advierten sobre la oferta de alternativas de trabajo como lo es la naturaleza interdisciplinaria, conjunto de conceptos, principios, técnicas, conocimientos, y los enfoques eclécticos de diferentes escuelas, siendo las piezas básicas para que el administrador pueda dirigir operacional y efectivamente su organización. Por tanto, el estar conciente de la importancia de los procesos operacionales, le ofrece estructura administrativa a ésta, donde cada miembro conoce e internaliza la importancia de sus funciones dentro de la organización.

Dentro de los enfoques y teorías, Drucker (2003) analiza que las organiza- 
ciones se deben dirigir mirando siempre hacia el futuro en alianzas, flexibilidad de trabajo y con liderazgo futurista en los administradores y demás personal.

De esta manera, se pueden identificar las características más resaltantes de un nuevo paradigma organizacional, las cuales pueden permitir transformaciones en las estructuras y maneras de actuar dentro de las instituciones en el mundo empresarial actual:

a) La organización se humaniza y es agente moral.

b) Es flexible, integral y abierta de acuerdo a sus finalidades.

c) Está centrada en el ser humano, el cual es considerado como un ser útil, concreto y ético.

d) La organización y el hombre giran alrededor del concepto de calidad humana, calidad orientada hacia un plan social donde se proyecta la vida buena.

e) Incorpora el concepto de endocalidad, el cual hace énfasis en el desempeño de la gente (Martín, 2000). Este concepto implica que el ser humano es visto como un ser físico, psicológico y espiritual dentro de su dimensión social.

Dentro de este marco contextual, hay un componente básico, novedoso, que avanza y transforma el dinamismo organizacional de las instituciones, es la información rápida y oportuna alcanzada a través de la tecnología y comunicación (Martín et al, 1997). Ello permite movilidad y acción en el devenir institucional, pero con criterios de responsabilidad y compromiso para mantener la presencia del hombre como el centro de desarrollo empresarial.

\section{Los nuevos senderos de la Universidad}

Los retos que deben enfrentar la sociedad y todas sus instituciones en este siglo XXI han sido objeto de atención y estudio, siendo abordados en todas sus vertientes, política, económica, social, educativa, valores, debido a la trascendencia de los hechos y fenómenos desarrollados en el contexto, los cuales inciden en la conformación de un nuevo orden mundial, siendo la Universidad un espacio que no escapa a esta realidad (Morin, 1999a).

Organismos internacionales, nacionales, entes gubernamentales y no gubernamentales, tal como se desprende de la United Nations Educational Scientific and Cultural Organization (UNESCO) en su cita de Declaratoria Mundial sobre la Educación Superior en el siglo XXI (1998), así como el propio seno de las Universidades en Latinoamérica han percibido los principios sustentados en esta declaratoria, en el interior de su organización, los cuales se presentan como la necesidad del logro de un verdadero sentido de su responsabilidad hacia lo humano y ético.

En este sentido, la convocatoria de la UNESCO en el año 1998 tuvo como acierto decretar la educación como uno de los pilares fundamentales de los derechos humanos, la democracia, el desarrollo sostenible y la paz, por lo cual deberá ser accesible para todos, a lo largo de toda la vida, y la necesidad de implementar medidas para asegurar la coordinación y cooperación entre los diversos sectores y dentro de cada uno de ellos y, en particular, entre la educación general, técnica y profesional secundaria y 
postsecundaria, así como entre universidades, escuelas universitarias e instituciones técnicas.

En este contexto, la solución de los problemas que se plantean al respecto en los albores del siglo XXI, estará determinada por la amplitud con miras a la sociedad del futuro, y por la función que se asigne a la educación en general y a la educación superior en particular, donde deben prevalecer los valores e ideales de una cultura de paz, debiéndose movilizar a la comunidad internacional con ese fin (UNESCO, 1998).

Asimismo, se acordó que la transformación y expansión sustanciales de la educación superior, la mejora de su calidad y su pertinencia, y la manera de resolver las principales dificultades que la asechan, exigen la firme participación no sólo de gobiernos e instituciones de educación superior, sino también de todas las partes interesadas, comprendidos los estudiantes y sus familias, los profesores, el mundo de los negocios y la industria, los sectores público y privado de la economía, los parlamentos, los medios de comunicación, la comunidad, las asociaciones profesionales y la sociedad.

Derivado de lo anterior, se exige que las instituciones de educación superior asuman mayores responsabilidades para con la sociedad y rindan cuentas sobre la utilización de los recursos públicos y privados, nacionales o internacionales (UNESCO, 1998).

A este respecto, Morin (1999b) tomando como escenario la convocatoria de la UNESCO, afirma que los sistemas de educación superior deberán aumentar su capacidad para vivir en medio de la in- certidumbre, para transformarse y provocar el cambio, para atender las necesidades sociales y fomentar la solidaridad y la igualdad; preservar y ejercer el rigor y la originalidad científicos con espíritu imparcial, por ser un requisito previo decisivo para alcanzar y mantener un nivel indispensable de calidad, y colocar a los estudiantes en el primer plano de sus preocupaciones en la perspectiva de una educación a lo largo de toda la vida, a fin de poder integrar plenamente en la sociedad mundial del conocimiento del siglo que viene, donde la cooperación y el intercambio internacionales son mecanismos decisivos para promover la educación superior en todo el mundo.

Igualmente, la Conferencia General de la UNESCO (1997), cuyo encuentro permitió trazar la recomendación relativa a la condición del personal docente de la enseñanza superior, el personal administrativo y los estudiantes universitarios, considera que éstos deberán cumplir con una función ética, autonómi$\mathrm{ca}$, responsable y prospectiva, en beneficio de las casas de estudio en las cuales se desenvuelven y de su sociedad. De ahí que será necesario en estas casas de estudio:

a) Preservar y desarrollar sus funciones fundamentales, sometiendo todas sus actividades a las exigencias de la ética y del rigor científico e intelectual.

b) Poder opinar sobre los problemas éticos, culturales y sociales, con total autonomía y plena responsabilidad, por estar provistos de una especie de autoridad intelectual que la sociedad necesita para ayudarla a reflexionar, comprender y actuar. 
c) Reforzar sus funciones críticas y progresistas mediante un análisis constante de las nuevas tendencias sociales, económicas, culturales y políticas, desempeñando de esa manera funciones de centro de previsión, alerta y prevención.

d) Utilizar su capacidad intelectual y prestigio moral para defender y difundir activamente valores universalmente aceptados, y en particular, la paz, la justicia, la libertad, la igualdad y la solidaridad, tal y como han quedado consagrados en la Constitución de la UNESCO.

e) Disfrutar plenamente de su libertad académica y autonomía, concebidas como un conjunto de derechos y obligaciones, siendo al mismo tiempo, plenamente responsables para con la sociedad, rindiéndole cuentas.

f) Aportar su contribución a la definición y tratamiento de los problemas que afectan al bienestar de las comunidades, las naciones y la sociedad mundial (UNESCO, 1997).

Con referencia a lo anterior, Morin (1999a) señala que la Universidad deberá adaptarse, simultáneamente, a las necesidades de la sociedad contemporánea y llevar a cabo su misión, transecular de conservación, transmisión y enriquecimiento de un patrimonio cultural, sin el cual la humanidad no sería más que máquina, produciría y consumiría.

Fundamentalmente, este enriquecimiento se traduce en una realimentación del vigor intelectual para enfrentar el conservadurismo académico, así como el derrumbe de los modelos y esquemas tradicionales enquistados en los espacios institucionales de la academia, los cuales abarcan desde el aspecto meramente organizacional hasta el gerencial, donde el aspecto humano y ético se ha puesto en observación.

Por tanto, se percibe en la sociedad una creciente preocupación por la definición de una nueva visión de la educación superior, traducida básicamente en una renovación del sistema por diferentes vías y mecanismos. Esencialmente, se aspira a un mayor nivel de pertinencia interna y externa de sus resultados, en correspondencia con los problemas fundamentales de las sociedades subdesarrolladas, que concuerda con Llanoz De la $\mathrm{Hoz}$ (2000), quien considera que los retos son de transformación de las estructuras organizacionales, por lo cual los centros de educación superior deberán mejorar su contexto interno-operativo, para avanzar a dimensiones humanas que serían cubiertas de una manera apropiadamente contextualizada en su espacio geográfico y de cara a los comportamientos de cada sociedad.

\section{La gerencia en las instituciones de educación superior}

El término gerencia tiene varias acepciones las cuales corresponden al área donde se esté ejecutando. Así, se habla de gerencia empresarial, gerencia de servicios públicos, gerencia del conocimiento, gerencia académica, gerencia universitaria. Pero, de igual manera, se emplea el término gestión de la educación superior, como equivalente a gerencia de las instituciones universitarias (Llanoz de la Hoz, 2000). 
Partiendo de este concepto, la gerencia de las instituciones de educación superior está asociada a la búsqueda de una mayor y mejor calidad de servicio universitario y una mayor calidad del desempeño de sus órganos y actores en el entramado institucional, con el fin de dar respuestas a las carencias y expectativas institucionales y de la sociedad en general.

Se requiere para la gerencia universitaria del dominio de diferentes áreas del conocimiento como son la administrativa, política, económica, legal y con particular énfasis en el conocimiento de la cultura organizacional específica.

Instituciones y actores del mundo académico, como por ejemplo, la Universidad Central de Venezuela (2002), definen la gestión académica como aquel proceso sistémico de planificación, ejecución y evaluación, en la docencia, investigación y extensión. La planificación debe hacerse en función de la misión de las Universidades y de la planificación estratégica de las mismas, visualizándose el problema de la gestión académica desde el proceso de admisión hasta el egreso. Mas sin embargo, la Universidad de Cali (2004) en la década de los '90 adelantó un ambicioso proceso de reforma curricular y de cambios de todos los procesos gerenciales, con el fin de concretar la idea de la formación integral.

No obstante, pese a la rectitud de los propósitos, no se aplicó de manera adecuada desde el punto de vista de los medios. No se hizo una evaluación de costos, de los impactos financieros de la misma o de la forma como esta reforma afectaba la estructura de la organización académica de la Universidad, lo cual condujo a hacer un planteamiento teórico de la gerencia universitaria, concibiéndola como el proceso por el cual se planifica, ejecuta y controla la vida académica en razón de sus medios y fines, pero atendiendo a las causas y efectos de todas las acciones.

Paralelamente, la Universidad del Valle (2003) inició un programa de revisión de las políticas académicas plasmadas en el Acuerdo 001 de 1993 del Consejo Superior, con el fin de redefinir sus fundamentos, estrategias y desarrollos programáticos y vida gerencial-administrativa. En esta labor, se planteó la gerencia universitaria, como la actividad que planifica, ejecuta y evalúa el encuentro de dos procesos contrarios y paralelos como es la academia y la función administrativa, de donde la primera señala un orden de resultados sociales, y la segunda un orden de exigencia de resultados financieros.

En torno a esto, la Universidad Autónoma de México (2002) señala que para poder cumplir con el gran reto académico del siglo XXI, debe redefinir su visión sobre la gerencia universitaria. En este sentido, el incremento de su población estudiantil pasa por un consenso ético de la vida financiera de la Universidad, en el cual se deberán sacrificar determinados factores, valiosos para la vida universitaria como los traslados de las autoridades a los diferentes eventos que se presentan, y en la cual debe hacer presencia la Universidad Autónoma de México, como primera casa de estudios de ese país, pero que impiden su crecimiento por la cantidad de recursos económicos invertidos en esas actividades.

Dentro de ese marco, Llanoz de la Hoz (2000) concibe la gestión universita- 
ria como un proceso que comprende determinadas funciones y actividades laborales de gestión, a fin de lograr los objetivos de la organización. En esa gestión, los directivos utilizan principios de carácter administrativo, que les sirven de guía en este proceso, pero, igualmente, permiten la articulación de todos los elementos presentes y aquellos necesarios para la toma de soluciones (Drucker, 2003).

Esos principios bien pudieran ser los elementos que conforman los nuevos paradigmas, donde la organización vertical, la cual genera resistencias, no tiene lugar, surgiendo la necesidad de administrar en forma horizontal (Koontz y Weihrich, 2002), mientras gana terreno una nueva cultura organizacional que impone la elaboración de una visión compartida y una perspectiva creativa e independiente en la cual haya un acercamiento entre los líderes y el personal, con una comunicación integral que incluya el aspecto técni$\mathrm{co}$, pero de igual manera los valores establecidos en la organización y los individuales (Drucker, 2003).

Del mismo modo, Muro (1998) imprime a la gestión universitaria un carácter constructivo y reconstructivo del sentido organizacional y de las relaciones de autoridad, sobre la base de principios y valores compartidos que incorporen mecanismos de participación en la toma de decisiones. Así, la gestión se traduce en un proceso global, complejo, integrador y generador que requiere de la conjunción de principios, modelos, estrategias, mecanismos y estilos gerenciales, con supuestos epistemológicos, ideológicos, axiológicos, con el fin de garantizar el crecimiento, fortalecimiento y desarrollo sostenible de la organización.
Sin duda, entender el concepto de gerencia dentro de las instituciones de educación superior, es un aspecto clave para poder señalar el cumplimiento de sus funciones sociales, luego ésta puede ser construida sobre la base de premisas o supuestos diferentes como el significado que tiene para las autoridades como gestores universitarios, la relevancia para los otros actores de la comunidad académica, así como las implicaciones que tienen estos conceptos para la construcción y avance de las instituciones de educación superior.

\section{Las dificultades en la gerencia universitaria}

El concepto de Gerencia de la Educación Superior plantea una dificultad inicial en relación con su alcance y sentido. Desde el punto de vista general, diferentes autores lo describen como la conjunción de procesos de organización y dirección en el ámbito educativo superior, la distribución y ejercicio de la autoridad y la relación entre el conjunto institucional y los niveles de gobierno real o racional (Díaz et al, 1997; Lanz, 2001 y Fuenmayor, 2001).

Tal y como lo señala Fuenmayor (2001), resulta importante considerar en el tema de la gestión universitaria, el significado especial de la variable política, particularmente en los procesos de transición democrática, que demandan la existencia de mecanismos eficaces de consenso y legitimación. Pero, otro factor crítico, de acuerdo a lo expresado por Lanz (2001) es el relativo a las formas de gobierno, bien sean colegiadas o jerarquizadas. Éstas, son objeto de análisis 
permanente, con el fin de replantear estructuras y procedimientos que contribuyan de manera efectiva a la integración de las funciones sustantivas de la Educación Superior.

No obstante, aspectos referentes al funcionamiento, como el relacionado con la coordinación institucional, resulta significativo condicionarlo por la especificidad del trabajo académico y sus formas de organización; de ahí, que el diseño de políticas y su efectiva coordinación requiere de acuerdos mínimos con los distintos actores de la comunidad académica (Koontz y Weihrich, 2002).

Con relación a la gestión en instituciones de educación superior, las transformaciones políticas y sociales del Estado han favorecido en su área administrativa o gerencial una dirección colegiada, asegurando la participación de los diversos actores en situaciones específicas, en la cual la centralización y la descentralización son consideradas como procesos contradictorios entre sí, pero que conducen a una maduración de las instituciones, siendo la última de éstas la que adquiere mayor fuerza en la toma de decisiones, luego propicia la autonomía de funciones y se hace responsable con el compromiso alcanzado socialmente (Martín et al, 1997).

Sin embargo, es importante señalar que debe imperar un equilibrio armónico en el seno de las Universidades entre centralización y descentralización para facilitar el manejo de la complejidad y la incertidumbre, rasgos presentes en la gestión universitaria. Al respecto, Koontz y Weihrich (2002) se refieren a la centralización como el grado organizacional en el cual la toma de decisiones se concen- tra en un solo punto de la organización a diferencia de la descentralización, la cual se presenta cuando hay aportes del personal de nivel inferior o se le da realmente la oportunidad de ejercer participación en la toma de decisiones.

Atendiendo a estas consideraciones, en una organización descentralizada se pueden tomar acciones con mayor rapidez para resolver problemas, es decir, hay mayor aporte de información, luego el personal tiene la posibilidad de hacerlo, disminuyendo los márgenes de incertidumbre o riesgos a una decisión equivocada que puede causar un daño, en algunos casos irreparable.

Lo anterior conduce a precisar que en las Casas de Estudios Superiores de acuerdo a la Ley de Universidades (1967), las funciones académicas están plenamente regladas y en ningún momento pueden ser desviadas en su accionar; es decir, la norma de la materia regula de manera directa las acciones, procesos, procedimientos, las cuales en su mayoría son aquellas de carácter académico, y por tanto, susceptibles a una autoridad centralizada.

Otro aspecto importante reseñado por Fuenmayor (2001), el cual debe ser tomado en consideración en la gestión universitaria, alude a los procesos de evaluación y autoevaluación de las diferentes dimensiones y actores del tejido institucional universitario, orientado al mejoramiento continuo de los procesos, productos e impacto. Aspectos como la pertinencia, calidad, eficacia, productividad y rendimiento están asociados a la efectividad, claridad y autenticidad de un sistema permanente de evaluación, con 
énfasis en la dimensión cualitativa del proceso.

Esto conlleva a repasar los factores reseñados por Díaz et al (1997), Lanz (2001) y Fuenmayor (2001), como elementos vitales en la gestión universitaria, los cuales son:

a) La globalidad, al considerar importantes todas las partes constitutivas del mundo institucional.

b) La contextualización, necesaria y enmarcada en un tiempo y espacio determinado, así como el reconocimiento de diferentes planos: políticos, sociales, económicos, ecológicos, tecnológicos, que inciden en el funcionamiento institucional.

c) La complejidad, referida a la multidimensionalidad, multicausalidad, contradicción y mundo de incertidumbre que determina el intercambio de relaciones en el entramado institucional.

d) La democratización, relacionada con los procesos de participación, relaciones de poder, rendición de cuentas, negociación y concertación de las diversas fuerzas.

e) La ética, como proceso reflexivo relacionado con el mundo de las virtudes propias de cada individuo y su forma de mediar en los diferentes encuentros; la ética asociada a la valoración de la condición humana desde un clima de respeto y reconocimiento del otro desde el propio yo.

Acerca de este último aspecto, el de la ética como elemento presente en la gestión universitaria, Lanz (2001) lo concibe como un componente integrador en la conducción de la academia, pero además, contribuye a impedir la búsqueda de beneficios personales, procurando al mismo tiempo el florecimiento de los beneficios institucionales universitarios.

Por tanto, se impone una racionalidad individual por encima de la racionalidad institucional, una concepción de evaluación que considere toda una perspectiva ética inscrita en una lógica distinta a la tradicionalmente concebida para evaluar la calidad (Cortina, 1999); es decir, un criterio que contemple beneficios institucionales, pero partiendo de considerar a todas las partes del sistema sin exclusión, y en el uso de los recursos de manera homogénea y en sí de todos los elementos que conforman los puntos clave cuali-cualitativos de la gestión en el mundo universitario.

\section{La ética: una vía de crecimiento organizacional}

No es suficiente hacer referencia al compromiso que pueda tener la Universidad dentro y fuera de sus componentes organizacionales. No es suficiente analizar el rol protagónico ni el trabajo realizado por sus directivos, si dentro del clima organizacional donde ellos actúan, no se le da cabida a la ética.

La ética ha sido siempre un tópico importante de discusión y conversación a lo largo de toda la historia de la humanidad. Son muchos los temas que emergen de ella, pues el hombre en esencia lleva dentro de su devenir social un cúmulo de pasiones y necesidades que desbordan en un sentido de existencia moral, de virtudes y bondades, de maldades y defectos. Por ende, puede decirse que hay un juego de haberes y voluntades dentro del mundo del ser en existencia. 
En ese sentido, Morin (1999b) concibe cuatro dimensiones de la experiencia humana en el devenir social y organizacional, consideradas como las claves para lograr la felicidad en el trabajo y proyectar una excelencia corporativa sostenida y constante, estas dimensiones son: a) la dimensión intelectual, que aspira a la verdad; b) la dimensión estética, que aspira a la belleza; c) la dimensión moral, que aspira a la bondad, y d) la dimensión espiritual, que aspira a la unidad.

Verdad, belleza, bondad y unidad son los elementos que estructuran la vida humana, por lo tanto, no deben ser omitidos en el quehacer organizacional de las instituciones, y, generalmente, el trabajo institucional se cumple omitiendo lo humano como dimensión existencial, sólo interesan los logros materiales y financieros, sólo se observan los resultados y no la constante del desempeño laboral, la cual debe puntualizar en la excelencia humana a través de la verdad, la belleza, la bondad y la unidad (Morin 1999a).

Por su parte, Martín (2000) señala que definir la ética requiere ubicarla desde diversos puntos de vista. Etimológicamente, ética, viene del griego "ethos", significa costumbre y por eso se ha definido como la doctrina de las costumbres. Pero de igual manera, se le considera en su significado como, morada, hábitat, carácter, modo de ser. En este sentido, el concepto ha evolucionado y hoy se le reconoce como un conjunto de valores, como una posibilidad del hombre para alcanzar sus metas, movilizando sentimientos y voluntades (Brow, 1998).

Para Aristóteles (2001), las virtudes éticas son aquellas que se desen- vuelven en la práctica y van encaminadas en la prosecución de un fin, vinculadas al concepto de vida práctica, a un saber práctico donde el hombre en tanto ser social muestra acciones, puede elegir y demostrar conductas éticas y morales.

Cuando se habla de ética, no se hace referencia a normas externas impuestas a organizaciones, instituciones, oficios, empresas o individuos. La moral no proviene de afuera, es un conjunto de convicciones internas que inducen a un rendimiento y a una eficacia vital a cada uno de ellos (Cortina, 2000).

Resulta importante señalar que ética y moral se han venido usando indistintamente en la práctica, dada su dependencia recíproca. En este caso, la ética es distinta a la moral, siendo que Sánchez (2002) señala a esta última, como un sistema de normas, preceptos y deberes que regulan los actos humanos individuales y sociales en función a la bondado malicia de los mismos.

Sobre este particular, Cortina (2000) sostiene que la ética tiene por objeto el estudio y análisis de la moral, no la hace ni la predetermina, muy por el contrario, se edifica a partir de la moral, surge del análisis y reflexión sobre la praxis, en fin, se basa en la experiencia moral.

Concebida la ética como una acción con dimensión externa o interna, ésta engloba a su vez la dimensión personal, construida por el individuo al recibir de la ética cívica, los valores morales compartidos, los cuales permiten avanzar a las sociedades, luego la ética moderna se desarrolla con una función integradora (Martín, 2000). En virtud de esto, Sánchez (2002) la identifica como la racionalización del comportamiento huma- 
no, mediante principios y normas, basadas en valores universales, que buscan el desarrollo óptimo para el hombre como individuo y como grupo, donde impere la verdad, justicia y bondad.

Por otra parte, Cortina (2000) plantea que está presente la ética aplicada, aquella que transforma y revitaliza las instituciones modernas, generando cambios positivos en la empresa o institución y, en última instancia, en la sociedad general.

Adicionalmente, Martín (2000) señala dentro de la clasificación para la puesta en escena de estudios y enfoques acerca de la ética, a la metaética, la cual estudia los principios de la ética, sus orígenes, significados y alcances.

Según Cortina (2000), también se encuentra la ética normativa, cuyo propósito es establecer estándares y pautas para la conducta moral, dentro de regulaciones sociales, y por último, se debe mencionar la ética aplicada, la cual permite una reflexión ética sobre problemas controversiales, moralmente significativos y prácticos.

En la ética aplicada se ubica la ética de las organizaciones, la cual está relacionada con la conducta humana en el campo social y cultural, tales como: la médica, la empresarial, la sexual, la social, la educativa, entre otras. Por lo tanto, la ética se hace un saber práctico, estrictamente humano.

Agrega este autor, que el concepto de ética debe estar vinculado al concepto de vida práctica, de la vida moral como un saber encomiado de los principios, valores y morales del hombre; además, la ética hace énfasis en la responsabilidad y en las conductas, las cuales deben ser comunicadas, deliberadas, discutidas para llegar a acuerdos con resultados y decisiones acertadas dentro del contexto organizacional, situación que casi nunca se establece en las instituciones universitarias.

Por el contrario, en los centros de educación superior se destaca la imposición de normas y reglamentos generalmente no asimilados ni cumplidos por el personal, como una manera de sentir los procesos bajo un compromiso de conciencia y constancia, de acciones demostradas con responsabilidad, comunicación, orientación y acuerdos, conduciendo al establecimiento de una organización de convivencia (Morin, 1999a).

Todo lo anterior, lleva a enfocar la ética como un tipo de saber práctico preocupado por averiguar cuál es el fin de las acciones, para así decidir qué hábitos pueden asumirse, cómo ordenar las metas intermedias, cuáles son los valores que sirven de orientación, qué modo de ser o carácter van a ser incorporados, a fin de obrar con prudencia, es decir, tomar decisiones acertadas (Cortina, 2000).

Sin duda alguna, esta definición que hace la autora describe la práctica, la acción, los hábitos, las metas, los valores, el modo de ser, la prudencia y la decisión acertada, como una vía sencilla de actuar en la vida para alimentar la razón de permanencia a través de una vida útil dentro de espacios compartidos.

Por tanto, el concepto de ética debe estar destinado hacia la acción, es decir, hacia la conducta, ya que la norma o el código no señalan las actitudes de las personas, ello surge de la combinación de estos factores, pero con la ética marcando la integración de los mismos. La 
ética se logra por medio de un proceso de formación social sostenida y activa, del día a día, donde los valores sean representados dentro de una práctica observable, tangible y dispuesta a mejorar las condiciones de vida social.

En ese sentido, la ética no puede ser considerada como un conjunto de reglas y castigos; más bien, debe ser un proceso para decidir lo que debe hacerse, y así tener un sistema justo que respete los derechos donde los grupos tengan el poder para hacer lo correcto (Brown, 1998).

Las organizaciones, como parte de la sociedad, están llamadas a vincularse, cada vez más, a las exigencias que la misma requiere. Sociedad, organización e individuo deberán mantener un equilibrio de crecimiento y desarrollo que permita dar una imagen de integración, cooperación, colaboración y formación del individuo, en tanto ser físico, espiritual y moral.

Sobre la base de estos conceptos, Cortina (2000) hace énfasis en que las organizaciones requieren una misión y una visión dentro de su cultura y dentro de la sociedad. Esto se logra apuntalando el trabajo dentro de una perspectiva de ética aplicada, como debería ser toda ética de las organizaciones y de profesiones, en el sentido, no sólo de aplicar los principios generales, sino averiguar a la vez, cuáles son los bienes internos que cada una de las actividades deben proporcionar a la sociedad, qué metas debe perseguir y qué valores y hábitos incorporar para alcanzarla.

En todo caso, la gerencia es el lugar de decisión donde se orienta la acción. Es el proceso de hacer que las acti- vidades se cumplan con eficiencia y eficacia por medio de otras personas (Robbin y Coulter, 2000). En razón de esto, la función gerencial se orienta de una manera direccional y directorial, es decir, que señala la orientación a la meta y dirige el proceso para alcanzarla (Díaz, 2000).

Es precisamente en la gerencia -como señala Díaz (2000)- donde se toman las decisiones, trayendo consigo la deliberación, la preferencia, la valoración; es allí donde reposa la responsabilidad, la cual dibuja en ese momento con quién y hacia quién dirige la acción de la misma toma de decisiones. Es aquí donde coinciden la ética y la gerencia, precisamente en la responsabilidad social.

Si bien es cierto, se está transitando y viviendo en la sociedad de las organizaciones, donde la empresa constituye el paradigma de todas las restantes, en ese sentido la organización debe ser y actuar para contrarrestar los defectos y problemas de la viejas instituciones, considerando en su quehacer las siguientes características: ser éticamente impecable, es decir, cumplir estrictamente con los elementos éticos, satisfacer las necesidades humanas, contar con la agilidad y la iniciativa, determinando que a todos los mueve un interés común, además de tener presentes la solidaridad, el riesgo razonable y la corresponsabilidad de todas las acciones.

Esto lleva a pensar que las organizaciones están marcadas por el liderazgo-directivo; en consecuencia, un directivo que ejerza un liderazgo integral, debe tener ciertas características que lo identifiquen. Según Quinn (1996), existe un modelo de "competencia de valores", el 
cual relaciona los roles del liderazgo directivo con sus respectivas competencias bajo un enfoque integral. Resulta interesante este modelo porque orienta el trabajo directivo, de manera tal, que se ocupa de los diferentes campos organizacionales. Los roles serían, según este autor, el de coordinador, promotor, agente, director, innovador, productor, monitor $y$ facilitador.

Además de estos roles y sus competencias, Cortina (1999) identifica entre las cualidades del directivo: saber actuar, tener agilidad para hacerlo, demostrar la capacidad para proyectar e ilusionar con sus proyectos, además de poseer la habilidad para colocar a los miembros de la empresa en el lugar oportuno; la imaginación, la capacidad de innovar para adaptarse mejor a una realidad social siempre cambiante; reconstruir la legitimidad de la organización; comprender la organización como un proceso organizativo; poner énfasis en la toma de decisiones; interrelacionar un conjunto de elementos que configuran el proceso de constitución de la organización y la comprensión de sus finalidades como clave para comprender los procesos.

Con esta caracterización puede orientarse una discusión reflexiva para la formación de un componente de gestión dentro de un saber práctico, éticamente comprometido con la institución, asociado a la vida personal, profesional y organizacional como un punto de acción de la vida social.

Todo conlleva a que las universidades venezolanas, en el ejercicio constante de sus funciones, operaciones, procesos, puedan adecuar su visión en razón de su contexto y, en consecuencia, ejecutar sus estrategias incorporando la responsabilidad social como factor clave del éxito y como elemento ético, donde la justicia en la organización sea el punto de apoyo de gestión y toma de decisiones.

\section{Consideraciones Finales}

El papel de la universidad venezolana en este momento de cambio necesario y fundamental, representa una nueva expresión de la forma de vida del universitario, lo cual resulta una responsabilidad ineludible y prioritaria como venezolanos.

Ciertamente recobrar el sentido y el valor de la educación en Venezuela, implica asumir con verdadero compromiso la acción de cambio y renovación que debe imperar, al determinar de forma cierta, en este caso, la sociedad que se espera en este siglo XXI, ello implica naturalmente el rompimiento de privilegios y paradigmas que han campeado en el quehacer universitario, y que por su propia naturaleza atentan contra lo que debe reinar en las casas de estudios universitarios.

La universidad venezolana, en su universalidad es de todos los venezolanos, y en este orden de ideas, debe contribuir al sostenimiento y desarrollo eficiente de las aspiraciones, lo que en sí es buscar y lograr la calidad de vida. En este sentido, la relación entre calidad de vida y desarrollo educativo, dejará de ser una relación técnica y de expertos para convertirse en el vocabulario común de los universitarios, quienes con sus manos hacedoras y creativas contribuirán final- 
mente al florecimiento de la Patria que se ha soñado.

Es vital comprender la estrecha relación de una buena educación con la formación de ciudadanos autónomos, informados, comprometidos, responsables y tolerantes, con el conocimiento, valores y habilidades para adaptar o crear innovaciones tecnológicas; con la habilidad de razonar y la capacidad de aprender por su propia cuenta, se apoyará lo imprescindible como es formar personas inteligentes y productivas.

Una educación socialmente equitativa, contribuye a la integración, estabilidad social, a la armonía, capacitación de la fuerza de trabajo y a la formación de una comunidad de ciudadanos informados y responsables. También promueve la tolerancia política, reduce la violencia $y$, por lo tanto, crea un clima más favorable para las inversiones nacionales e internacionales y para el crecimiento y prosperidad social y cultural.

El gran desafío de la Universidad venezolana es proveer educación de calidad para enfrentar el desarrollo de un país que lo exige. Esto implica, por supuesto, realizar inversiones sustanciales y renovar esfuerzos para mejorar la gestión educativa y los niveles de formación y remuneración, promover la reforma curricular y desarrollar nuevos materiales de enseñanza, utilizando los medios tecnológicos de información y comunicaciones que se disponen en la actualidad, empleando esencialmente los recursos financieros, humanos y técnicos de manera adecuada, es decir, profesional y éticamente.

La ética y la educación están emparentadas por sus fines: el crecimiento y el crecimiento pleno de las personas. EI quehacer esencial de la Universidad es proporcionar un ambiente que favorezca el desarrollo y realización de todos sus integrantes y a través de ellos beneficiar a la sociedad en general.

La ética universitaria o la ética en la Universidad, tiene a su cargo mostrar los medios y elementos más convenientes y debidos para la construcción de ese ambiente y el cumplimiento de sus fines. La primera prescripción de la ética a la Universidad es su compromiso con la verdad y la justicia en el ejercicio del pensamiento. Ya ésta es una indispensable tarea. Así como todo el sistema filosófico se inicia con el problema de la verdad, de la legitimación del conocimiento para conocerla, así la Universidad abre las puertas que conducen al mundo de verdades.

Éste es verdaderamente el deber ético más importante de la Universidad: mostrar, iluminar a los estudiantes, luego es excelente obligación ética de la Universidad: demostrar que las sombras son sombras, y hay que recorrer el camino hacia la luz y así enfrentar a las sombras de las cavernas.

Este deber de la Universidad es necesario, pero también difícil y arriesgado, porque los guardianes y constructores de las sombras y los custodios de las cavernas son poderosos y su poder se sustenta en el reino de la dominación y la ignorancia.

Hay algunos medios para la formación de esta conciencia ética en la Universidad. Por la esencia del quehacer universitario, la evidencia intelectual ha de ser el medio más poderoso. Un medio indispensable para esto, es la práctica de la justicia institucional. Una Universidad forma hábitos, actitudes justas, sobre todo si es una Institución donde reina la razón y se vive la justicia. 
La Universidad debe ser convertida en un lugar donde los estudiantes puedan encontrar los medios y oportunidades convenientes para su desarrollo personal en los planos intelectual, estético, espiritual y moral; donde los profesores investiguen, generen y comuniquen conocimientos, muestren caminos deseables con sus propias vidas, comuniquen a sus alumnos el placer del saber, pero también dispongan de posibilidades económicas, académicas y culturales para crecer y proyectar así su crecimiento.

Para esto es indispensable contar con funcionarios preparados para organizar, decidir, ordenar, innovar, gobernar, con interés por reflexionar y llevar adelante el destino de la Universidad de acuerdo a sus fines y medios en la prosecución de la justicia social, pero partiendo de la Universidad buena, justa, verdadera, es decir, ética.

Es bueno repetir que la Universidad ética, es la que enseña la justicia, la bondad, la verdad, pero siendo para esto justa. Es la Universidad justa la que está gobernada por criterios y lideres justos. Es este liderazgo el que empieza con un gobierno ético de verdad y, sobre todo, de acciones académicas que enseñen lo que es la vida dentro de una organización universitaria, y así, reflejen hacia la sociedad en la que está inmersa, que la formación en su seno está sustentada por un criterio de verdad, belleza, bondad, unidad humana y luz.

\section{Referencias Bibliográficas}

Aristóteles (2001). Ética de Nicómaco. México: Editorial Pentagrama.
Argyris, Carl \& Shön, David (2001). Organizational learning: A theory of action perspective. Massachussets (USA): Addison Wesley.

Brown, Pete (1998). Ética hacia la Empresa. Madrid: Editorial Tecnos.

Castells, Max (1999). Sociedad-Tecnología y Transformación. Madrid (España): Editorial Trotta.

Bueno, Enrique (2002). Organización de Empresas. Madrid (España): Ediciones Pirámide.

Cortina, Adela (1999). Ética de la Empresa. Madrid (España): Editorial Trotta.

Cortina, Adela (2000). Hasta un Pueblo de Demonios. Madrid: Editorial Taurus.

Desiato, Freddy y Guevara, Antonio (1998). Hacia la Nueva Organización. México: Editorial Diana.

Díaz Barrios, Jazmín (2000). La Ética en la Gerencia vista a través del desarroIlo organizacional. En: Revista Venezolana de Gerencia. Año 5. No. 10, p. 49-61. Vicerrectorado Académico. La Universidad del Zulia. Maracaibo, Venezuela. ISSN 1315-9984.

Díaz, Barlette; Casanova, Héctor José; Maldonado, Andrés y López, Roberto (1997). Financiamiento y Gestión de la Educación Superior en América Latina y El Caribe. Visión de América Latina y El Caribe. Tomo I. Colección Respuestas. Ediciones CRESALC/UNESCO. Caracas.

Drucker, P. (2003). La Organización del Futuro. México: Editorial Mc Graw-Hill. México.

Fuenmayor, Luis (2001). Proposiciones para la Nueva Ley de Universidades. Debate Abierto. No. 16. Caracas.

Gibson, James; Ivancevich, John y Donnelly, James (2001). Las organizaciones: comportamiento, estructura y procesos. Santiago de Chile: Mc Graw-Hill. 
Guiddens, Anthony (2003). Consecuencias de la Modernidad. Mexico. Editorial Planeta.

González, Jorge; Ramírez, José; Espinosa, José y Peralta, Gabriel (2002). Elementos para la Construcción de un Modelo de Gestión Académica en el Nivel Medio Superior y Superior. En: La Comunidad del Conocimiento. México: Plaza y Valdés Editores. p. 49-61.

Koontz, Harold y Weihrich, Heinz (2002). Administración. Una Perspectiva Global. México: Mc Graw-Hill.

Lanz, Rigoberto (2001). El Decálogo de la Universidad que Queremos. Maturín: Editorial Instituto Pedagógico de Maturín.

Ley de Universidades (1967). Caracas: Editorial Paraninfo.

Llanoz de la Hoz, Héctor (2000). Hacia una nueva gestión exitosa de las Universidades Nacionales. Caracas: Impresión Taller Editorial de la Universidad Experimental Simón Rodríguez.

Marsick, Victoria (2000). Empresas que Aprenden. Barcelona (España): Editorial Gedisa.

Martin, Federico (2000). Ética. Maracaibo: Ediluz.

Martín, Enrique; Valera, Marcos; Pérez, Berenice; León, Juan y Santos, Sussi (1997). Experiencias en la gestión y el financiamiento de la Educación Superior en Cuba. La Educación Superior en el siglo XXI. Visión de América Latina y El Caribe. Tomo II. Colección Respuestas. Ediciones CRESALC/UNESCO. Caracas.

Morin, Edgar (1999a). Los Siete Saberes Necesarios para la Educación del Futuro. Paris: UNESCO.
Morin, Edgar (1999b). Introducción al Pensamiento Complejo. Barcelona (España): Editorial Gedisa.

Muro, Xiomara (1998). Criterios e Indicadores de Evaluación en la Calidad de Gestión del Financiamiento en Educación Superior. Caracas: Editorial Universidad Pedagógica Experimental Libertador.

Quinn, Fredd (1996). Liderazgo y Acción. México: Editorial Prentice Hall.

Ramírez, José; González, Jorge y Soriano, Luis (2002). Los Modelos Educativos en el Umbral del Tercer Milenio. En: La Comunidad del Conocimiento. México: Plaza y Valdés Editores. p. 49-61.

Robbins, Stephen y Coulter, Max (2000). Administración. México: Editorial Prentice Hall Hispanoamericana.

Sánchez, Martín (2002). Ética. Valencia (Venezuela): Editorial Vadell Hermanos.

UNESCO (1997). Declaración sobre la Educación Superior de Dakar. Conferencia General. Paris: Edición UNESCO.

UNESCO (1998). Declaración sobre la Educación Superior en América Latina y El Caribe. Conferencia Mundial sobre la Educación Superior. Paris: Edición UNESCO.

Universidad de Cali. Rectorado (2004). Esferas de la Gerencia Universitaria. Cali (Colombia): Edit. UniverCali.

Universidad Autónoma de México (2002). Políticas de Crecimiento Universitario. México: Editorial UNAM.

Universidad del Valle (2003). Una Nueva Gerencia Académica. Cali (Colombia): Editorial Universitas.

Universidad Central de Venezuela (2002). Políticas Académicas de la UCV. Vicerrectorado Académico. Comisión Central de Currículo. Caracas: UCV. 